\title{
Association analysis and the clinical significance of BRAF gene mutations and ultrasound features in papillary thyroid carcinoma
}

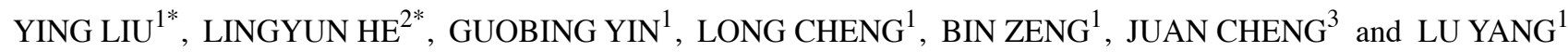 \\ ${ }^{1}$ Department of Breast and Thyroid Surgery, Second Affiliated Hospital of Chongqing Medical University, Chongqing 400010; \\ ${ }^{2}$ Department of Scientific Research and Education Section, Chongqing Health Center for Women and Children, \\ Chongqing 401120; ${ }^{3}$ Department of Ultrasound, Second Affiliated Hospital of Chongqing Medical University and \\ Chongqing Key Laboratory of Ultrasound Molecular Imaging, Chongqing 400010, P.R. China
}

Received June 9, 2018; Accepted June 14, 2019

DOI: $10.3892 / 01.2019 .10641$

\begin{abstract}
The aim of the present study was to evaluate the associations between the B-Raf proto-oncogene serine/threonine kinase (BRAF)V600E mutation and conventional and contrast-enhanced ultrasonographic features in patients with papillary thyroid carcinoma (PTC), and to subsequently investigate the clinical value of these associations. In total, 207 thyroid nodules (diameter $\leq 2 \mathrm{~cm}$ ) were selected. Conventional ultrasound, contrast-enhanced ultrasound, BRAFV600E mutational analysis and ultrasound-guided fine-needle aspiration biopsy were preoperatively performed, and histopathological assessment of PTC was postoperatively confirmed. The nodules were divided into 2 groups based on the BRAFV600E mutational analysis, namely the mutant or the wild-type variant groups, and the association analyses of the ultrasonographic features between these 2 groups were performed. Overall, 74.9\% (155/207) of the PTC nodules had the BRAFV600E mutation, while $25.1 \%$ (52/207) had the wild-type BRAF allele. The 2 groups were analyzed using univariate logistic regression analysis, which demonstrated no significant differences regarding morphology, boundary, hypoechogenicity of the nodules, blood flow signal, enhancement uniformity, enhancement degree and clearance time
\end{abstract}

Correspondence to: Dr Lu Yang, Department of Breast and Thyroid Surgery, Second Affiliated Hospital of Chongqing Medical University, 74 Linjiang Road, Chongqing 400010, P.R. China E-mail:24930208@qq.com

Dr Juan Cheng, Department of Ultrasound, Second Affiliated Hospital of Chongqing Medical University and Chongqing Key Laboratory of Ultrasound Molecular Imaging, 74 Linjiang Road, Chongqing 400010, P.R. China

E-mail: tenderrill@163.com; 93409108@qq.com

*Contributed equally

Key words: two-dimensional ultrasound, contrast-enhanced ultrasound, papillary thyroid carcinoma, BRAFV600E mutation
$(\mathrm{P}>0.05)$. Moreover, the 2 groups demonstrated significant differences regarding the aspect ratio, microcalcification, nodule size following enhancement, enhancement mode and enhancement time $(\mathrm{P}<0.05)$. A multivariate logistic regression analysis was performed to further validate the association of these features with the BRAFV600E mutation; however, only microcalcification [odds ratio (OR), 2.256; 95\% confidence interval (CI), 1.160-5.500; $\mathrm{P}=0.020]$ and nodule size following enhancement (OR, 2.119; 95\% CI, 1.039-4.321; $\mathrm{P}=0.039$ ) were associated with the BRAF mutational status. The associations found between the two ultrasonographic features and BRAFV600E mutation indicate that they can predict the BRAF mutational status to provide a reliable basis for clinical diagnosis and treatment.

\section{Introduction}

The incidence rate of thyroid carcinoma has increased markedly by 4.73 times from $2.40 / 100,000$ in 2003 to $13.75 / 100,000$ in 2012, with an average annual increase of $20 \%$ in China (1). Papillary thyroid carcinoma (PTC) is one type of thyroid carcinoma, which is fast growing (2). The majority of PTCs have low invasiveness and a good prognosis, but a number of tumors exhibit high invasiveness, mainly manifesting as lymph node metastasis, outer capsule invasion and distant metastasis. The B-Raf proto-oncogene, serine/threonine kinase (BRAF)V600E mutation is a common mutation found in papillary carcinomas (3). Multiple studies have revealed that BRAF mutations are associated with the invasiveness of PTC (4-6). Conventional ultrasound and contrast-enhanced ultrasound are important methods for diagnosing thyroid nodules. The present study aimed to analyze the associations between the ultrasound features of PTC and the BRAFV600E mutation and to determine whether ultrasound can facilitate a preliminary prediction of the invasiveness of PTC, which can assist clinicians by providing alternative choices (either observation or surgery). If these features of conventional and contrast-enhanced ultrasound are associated with BRAF gene mutations, despite a diagnosis of the thyroid nodule as PTC, BRAF gene analysis should be performed to stratify the risk of progression and guide clinical treatment (7). 


\section{Materials and methods}

Patients. All patients were examined and treated at the Second Affiliated Hospital of Chongqing Medical University (Chongqing, China). A total of 201 patients with thyroid nodules were examined with conventional ultrasound and contrast-enhanced ultrasound between October 2016 and April 2018. The patients included 62 males and 139 females, with ages ranging from 23 to 59 years, and a mean age of $44.58 \pm 7.684$ years. A total of 6 patients had 2 thyroid nodules each; therefore, 207 nodules were included in this study. Following examination, ultrasound-guided fine-needle aspiration biopsy (FNAB) was performed on all of the nodules and the specimens were evaluated for the BRAFV600E mutation. Following resection, nodules were fixed with $10 \%$ formaldehyde for $6 \mathrm{~h}$ at room temperature, the $5 \mu \mathrm{m}$ thick paraffin-embedded sections, were stained with hematoxylin and eosin for $80 \mathrm{~min}$ at room temperature, and subsequently confirmed as PTC under optical microscopy (x400 magnificaiton).

Conventional ultrasound and contrast-enhanced ultrasound examination of thyroid nodules. The nodules were examined with a color Doppler ultrasound (Philips IU22, L12-5 line array probe, 5-12 MHz; Philips Healthcare), which revealed the shape, boundary, internal echo, aspect ratio, microcalcification of and blood flow to the nodules (Fig. 1). Contrast-enhanced ultrasound of the thyroid nodules was used with a color Doppler ultrasound (Philips IU22, L9-3 line array probe, 3-9 MHz, mechanical index 0.07; Philips Healthcare). A median vein within the elbow was selected, and subsequently an ultrasound contrast agent $[2.4 \mathrm{ml}$ SonoVue (Bracco Suisse SA), diluted with $0.9 \%$ saline to $5 \mathrm{ml}$ ] was rapidly infused into the vein. Subsequently, $5 \mathrm{ml}$ $0.9 \%$ saline was immediately injected. The entry of the SonoVue until its disappearance was observed.

Ultrasound-guided FNAB of thyroid nodules. The thyroid nodules were examined with ultrasound to determine the insertion site, angle and depth of the needle, while the patients were in a supine position with their back raised and their head tilted back. Following routine sterilization of the injection site, $1-2 \mathrm{ml}$ of $2 \%$ lidocaine was used for local anesthesia. The probe was fixed and pressurized, with the puncture point on one side of the probe, and the puncture route was set at $30-40^{\circ}$ to the long axis of the probe surface (Fig. 1). Once the fine needle was positioned inside the thyroid nodule, the needle was repeatedly inserted and rotated 10 to 20 times and subsequently quickly withdrawn. The specimen was fixed onto a slide and visually designated suitable for further analysis and microscopic observations, prior to delivery to the Department of Pathology of the Second Affiliated Hospital of Chongqing Medical University (Chongqing, China) for cytological examination [the slide was fixed in 95\% ethanol for $15 \mathrm{~min}$ at room temperature, stained with hematoxylin and eosin for $20 \mathrm{~min}$ at room temperature, and observed under light microscope ( $x 400$ magnification)], and subsequently forwarded to the Molecular Diagnostic Laboratory of the Second Affiliated Hospital of Chongqing Medical University for BRAFV600E mutation analysis.
Materials for BRAFV600E mutation detection. The following materials were used for BRAFV600E mutation detection: A human BRAFV600E mutation detection kit (cat. no. ADx-BR04; Amoy Diagnostics Co., Ltd.), containing Taq enzyme, reaction mixture (containing the specific primer, the bi-loop probe, $50 \mathrm{nM}$ dNTPs, $750 \mathrm{nM}$ magnesium chloride, ammonium sulfate and potassium chloride) and a positive control (a mixture of 300 copies $/ \mu 1$ of BRAFV600E mutation plasmid and $10 \mathrm{ng} / \mu 1$ of wild-type BRAF human genome), a nucleic acid extraction reagent (formalin-fixed paraffin-embedded DNA; cat. no. ADx-T101; Amoy Diagnostics Co., Ltd.), purified water (self-prepared), a mini centrifuge [WTL-6K; Cence, China (http://www.xiangyilxj. com/)] and patient thyroid nodule cytological samples (FNA sample).

Methods of BRAFV600E mutation detection. Each patient thyroid nodule cytological sample was tested and analyzed along with a positive and negative control (purified water). The reaction mixture was thawed at room temperature, mixed on a vortex for $15 \mathrm{sec}$ and subsequently centrifuged at $2,000 \mathrm{x} \mathrm{g}$ for $15 \mathrm{sec}$ at room temperature. The reaction mixture $(35 \mu \mathrm{l})$ was mixed with $0.4 \mu \mathrm{l}$ Taq enzyme and subsequently dispensed into a PCR tube (kept on ice). Following this, $5 \mu 1$ DNA sample (2-5 ng), $5 \mu \mathrm{l}$ positive control and $5 \mu \mathrm{l}$ negative control were added separately to each PCR tube. The PCR tubes were centrifuged at 2,000 $\mathrm{xg}$ for $1 \mathrm{~min}$ at room temperature and a thermocycler (CFX96; Bio-Rad Laboratories, Inc.) was used. The following thermocycling conditions were used for the quantitative PCR: Initial denaturation at $95^{\circ} \mathrm{C}$ for $5 \mathrm{~min}$; 15 cycles of $95^{\circ} \mathrm{C}$ for $25 \mathrm{sec}, 64^{\circ} \mathrm{C}$ for $20 \mathrm{sec}$ and $72^{\circ} \mathrm{C}$ for $20 \mathrm{sec}$; followed by an additional 31 cycles of $93^{\circ} \mathrm{C}$ for $25 \mathrm{sec}$, $60^{\circ} \mathrm{C}$ for $35 \mathrm{sec}$ and $72^{\circ} \mathrm{C}$ for $20 \mathrm{sec}$. Carboxyfluorescein (FAM) and hexachloro-flororescein (HEX) signals were detected at $60^{\circ} \mathrm{C}$ during the final set of cycling conditions. The $2^{-\Delta \Delta C q}$ method was used to quantify the relative amount DNA (8). If the Cq value of the FAM signal of the sample was $\geq 28$, the sample was considered negative for the BRAFV600E mutation; if the $\mathrm{Cq}$ value of the FAM signal of the sample was $<28$, the sample was considered positive for the BRAFV600E mutation, according to manufacturer's protocol.

Statistical analysis. Statistical analysis was performed using SPSS (v23.0; IBM Corp.). Univariate logistic regression analysis was performed to determine the ultrasound factors associated with the BRAF gene mutation, and the variables with statistical significance on the univariate analysis were analyzed by multivariate logistic regression analysis. A two-sided P-value of $<0.05$ was considered to indicate a statistically significant difference.

\section{Results}

Ultrasonographic features and univariate analysis of the BRAF gene mutation. The conventional ultrasonographic features of PTC include either isoechoic or hypoechoic nodules, regular or irregular morphology, a clear or unclear boundary, an aspect ratio $<1$ or $\geq 1$, the absence or presence of microcalcification, and a rich or poor blood flow signal (Fig. 2). 
A a

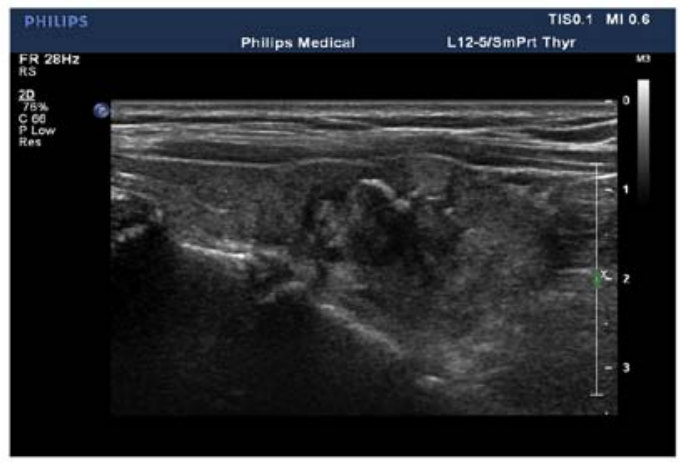

B

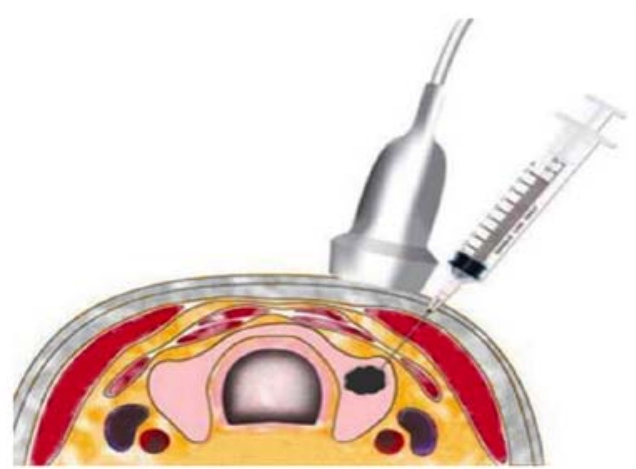

b

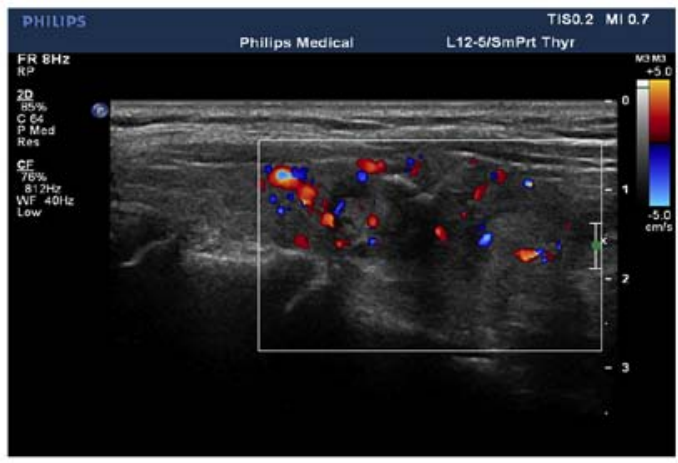

C

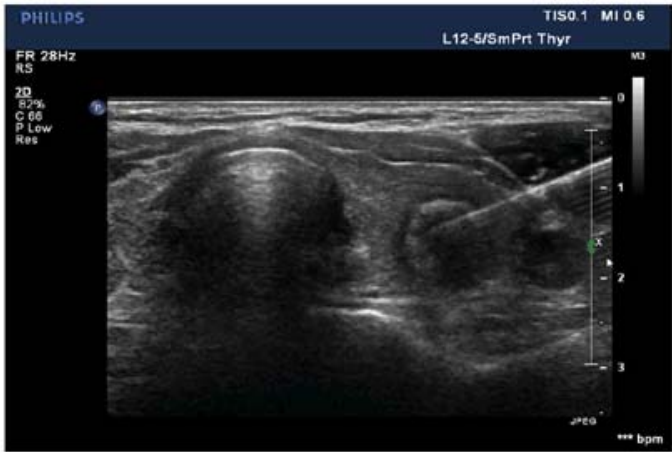

Figure 1. Ultrasound-guided fine-needle aspiration biopsy of thyroid nodules. (Aa and b) The thyroid nodule was examined with ultrasound, using (Aa) 2-dimensional grayscale ultrasound and ( $\mathrm{Ab}$ ) a color Doppler ultrasound. The red color in the center of the color Doppler ultrasound image indicates the blood flow signal of the nodule is toward the ultrasound probe, whereas the blue color indicates the opposite direction. The red and blue area on the right side of Ab indicates the blood flow range, which is the range of blood flow velocities that can be measured. (B) The puncture route is at $30-40^{\circ}$ to the long axis of the probe surface. (C) A fine needle was inserted into the thyroid nodule.

A a

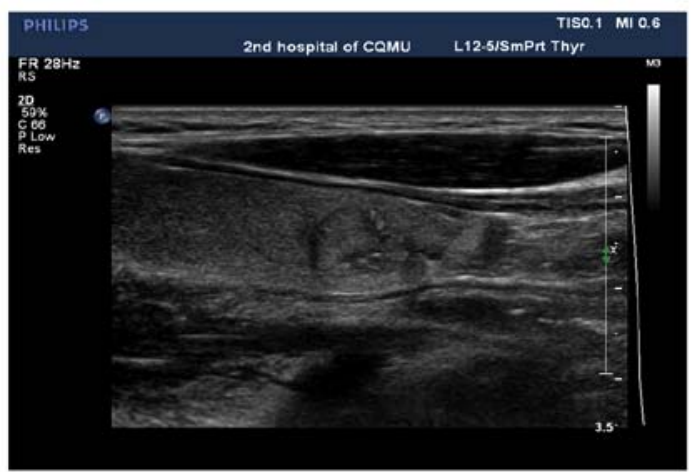

B a

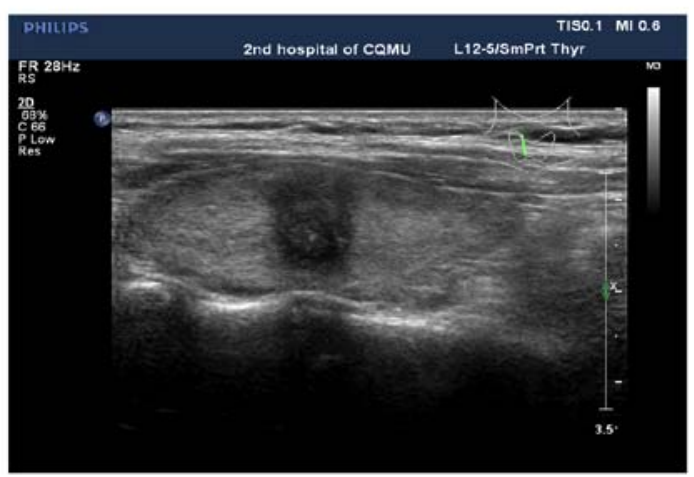

b

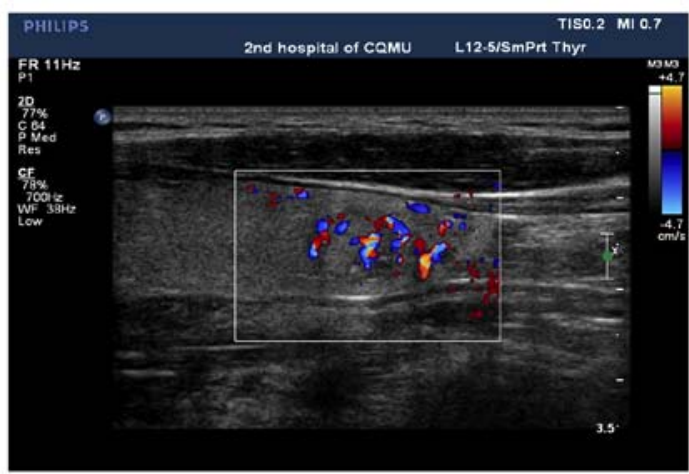

b

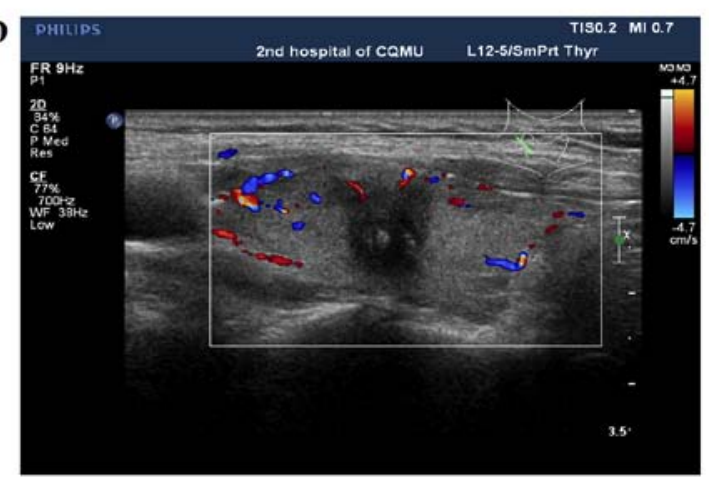

Figure 2. Conventional ultrasound of papillary thyroid carcinoma using (Aa and $\mathrm{Ba}$ ) 2-dimensional grayscale ultrasound and ( $\mathrm{Ab}$ and $\mathrm{Bb}$ ) a color $\mathrm{Doppler}$ ultrasound. (Aa and b) An isoechoic thyroid nodule with regular morphology, a clear boundary, an aspect ratio $<1$, the absence of microcalcification and a rich blood flow signal. (Ba and $\mathrm{b}$ ) A hypoechoic thyroid nodule with irregular morphology, an unclear boundary, an aspect ratio $\geq 1$, microcalcification and a poor blood flow signal. The red color in the center of the color Doppler ultrasound image indicates the blood flow signal of the nodule is toward the ultrasound probe, whereas the blue color indicates the opposite direction. The red and blue area on the right side of $\mathrm{Ab}$ and $\mathrm{Bb}$ indicates the blood flow range, which is the range of blood flow velocities that can be measured. Gray lines indicate the thyroid and the green lines indicate the position and direction of the ultrasound probe on the thyroid. 
A

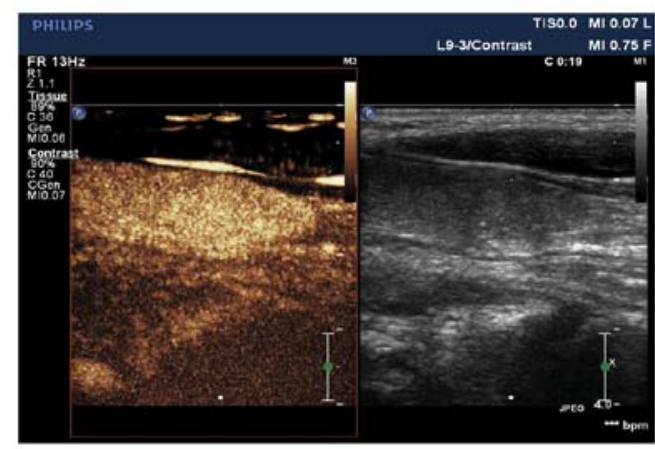

$\mathrm{C}$

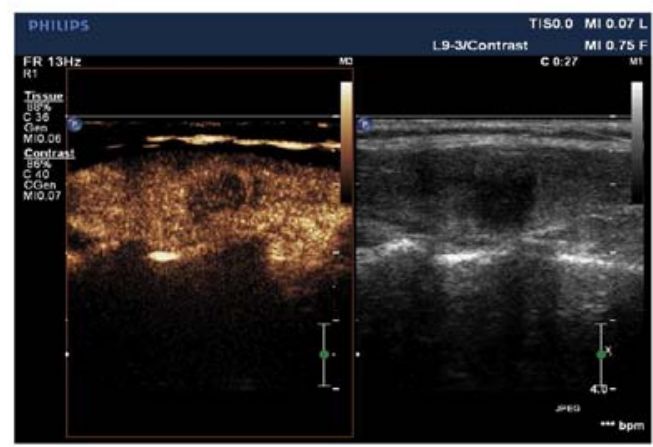

B
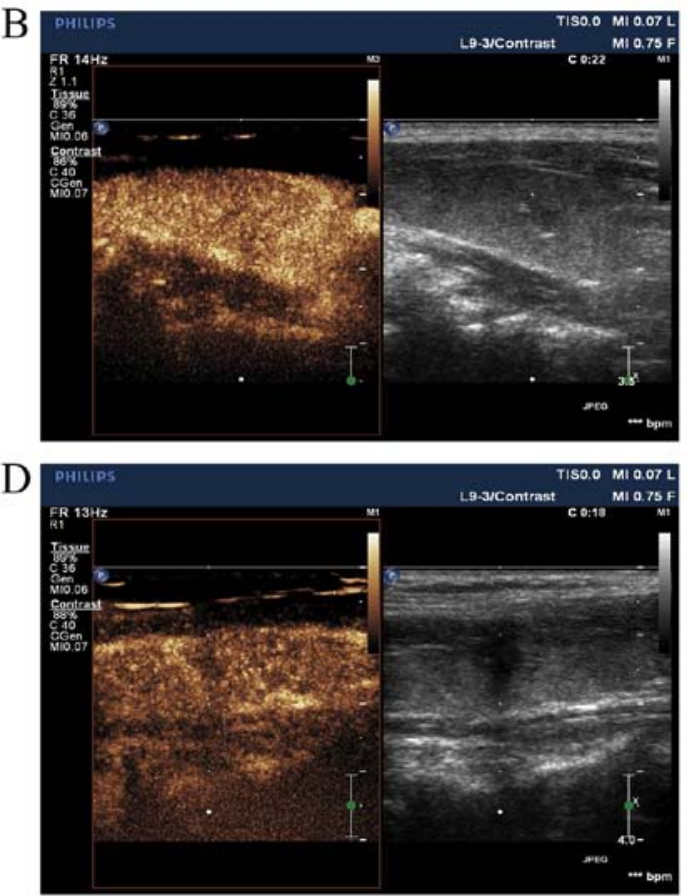

Figure 3. Contrast-enhanced ultrasonographic features of papillary thyroid carcinoma. (A) Homogeneous high enhancement of the nodule. (B) Homogeneous equal enhancement of the nodule. (C) Homogeneous low enhancement of the nodule. (D) Heterogeneous low enhancement of the nodule.

Contrast-enhanced ultrasonographic features of PTC are characterized by the following: Earlier/synchronous/ later enhancement of the nodule compared with that of the surrounding parenchyma, divergent/centripetal enhancement mode, homogeneous/heterogeneous high/equal/low enhancement at the peak (nodule contrast enhancement reached the peak), no change/increase in nodule size following angiography and earlier/synchronous/later clearance time (when SonoVue was completely cleared) to the surroundings (Fig. 3).

Overall, $74.9 \%(155 / 207)$ of the PTC nodules had the BRAFV600E mutation, while 25.1\% (52/207) had the wild-type BRAF allele. Conventional and contrast-enhanced ultrasonographic features of the nodules were univariately analyzed between the mutant and wild-type groups for the BRAFV600E mutation. There were a significantly higher number of nodules in the two categories with regard to the aspect ratio, microcalcification, nodule size following enhancement, enhancement mode and enhancement time in the mutant BRAF group compared with that in the wild-type BRAF group $(\mathrm{P}<0.05)$ (Table I).

Multivariate logistic regression analysis of ultrasonographic features associated with BRAF gene mutations. The variables with statistical significance in the univariate analysis were analyzed using multivariate logistic regression analysis and the BRAFV600E mutation was associated with microcalcification [odds ratio (OR), 2.256; 95\% confidence interval (CI), 1.160-5.500; $\mathrm{P}=0.020]$ and nodule size following enhancement (OR, 2.119; 95\% CI, 1.039-4.321; P=0.039) (Table II).

\section{Discussion}

With the continuous advancement of thyroid ultrasound diagnostic technologies and increasing attention to thyroid diseases, the incidence rate of thyroid cancer, particularly PTC, has been increasing over the last four decades (2). The majority of cases of PTC have good prognosis and the progression are slow therefore the patients could be monitored. The frequency of evaluation depends upon the sonographic features of the nodules, 6 to 12 months for subcentimeter nodules with suspicious characteristics, 12 to 24 months for nodules with low to intermediate suspicion on ultrasound, 2 to 3 years for very-low-risk nodules (9). The majority of thyroid nodules are stable during observation; however, a small number develop invasive manifestations, including tumor enlargement, lymph node or distant metastasis. The BRAF gene is a risk factor and may be associated with lymph node and distant metastasis (10). Preliminarily evaluation of the thyroid nodule to determine whether it is benign or malignant through non-invasive ultrasound examination would assist thyroid surgeons to provide patients with different treatment options.

The BRAF gene is a member of the RAF gene family and is located on chromosome 7q34. The BRAFV600E mutation is a substitution mutation within exon 15 at amino acid 600 , which changes to glutamic acid from valine and is one of the most common gene mutations in PTC (9). The prevalence of this mutation has been reported to be $40-70 \%$ (11-14). The BRAFV600E mutation promotes tumorigenesis and progression by increasing kinase activity and overactivating the mitogen-activated protein kinase pathway (15). The BRAFV600E mutation is associated with the invasiveness of PTC. Clinicopathological analyses have reported that PTC that has the BRAFV600E mutation is highly invasive and prone to capsular invasion, lymph node metastasis and a poor prognosis $(5,16)$. In the present study, the BRAF gene mutation rate was $74.8 \%$, which is consistent with previous reports (11-14).

PTC presents as unclear hypoechoic nodules on conventional ultrasound and is often accompanied by 
Table I. Association between ultrasonographic features and B-Raf proto-oncogene serine/threonine kinase gene mutation.

\begin{tabular}{|c|c|c|c|c|}
\hline Factors & Mutant $(\mathrm{n}=155)$ & Wild-type $(\mathrm{n}=52)$ & $\chi^{2}$ & P-value \\
\hline Morphology & & & 0.584 & 0.445 \\
\hline Irregular & 84 & 25 & & \\
\hline Regular & 71 & 27 & & \\
\hline Boundary & & & 0.849 & 0.357 \\
\hline Unclear & 80 & 23 & & \\
\hline Clear & 75 & 29 & & \\
\hline Hypoechoic nodule & & & 0.853 & 0.356 \\
\hline No & 72 & 28 & & \\
\hline Yes & 83 & 24 & & \\
\hline Aspect ratio & & & 5.216 & 0.022 \\
\hline$<1$ & 70 & 33 & & \\
\hline$\geq 1$ & 85 & 19 & & \\
\hline Microcalcification & & & 18.565 & $<0.001$ \\
\hline No & 57 & 37 & & \\
\hline Yes & 98 & 15 & & \\
\hline Blood flow signal & & & 0.129 & 0.719 \\
\hline Poor & 79 & 28 & & \\
\hline Rich & 76 & 24 & & \\
\hline Nodule size following enhancement & & & 11.660 & 0.001 \\
\hline No change & 62 & 35 & & \\
\hline Increase & 93 & 17 & & \\
\hline Enhancement uniformity & & & 1.004 & 0.316 \\
\hline Heterogeneous & 81 & 23 & & \\
\hline Homogeneous & 74 & 29 & & \\
\hline Enhancement degree & & & 1.169 & 0.280 \\
\hline Equal or high & 76 & 30 & & \\
\hline Low & 79 & 22 & & \\
\hline Enhancement mode & & & 11.614 & 0.001 \\
\hline Divergent & 51 & 31 & & \\
\hline Centripetal & 104 & 21 & & \\
\hline Enhancement time & & & 9.743 & 0.002 \\
\hline Synchronous or fast in & 57 & 32 & & \\
\hline Slow in & 98 & 20 & & \\
\hline Clearance time & & & 0.477 & 0.490 \\
\hline Synchronous or slow out & 89 & 27 & & \\
\hline Fast out & 66 & 25 & & \\
\hline
\end{tabular}

microcalcification, irregular morphology and an aspect ratio $\geq 1$. The diagnostic accuracy of conventional ultrasound for PTC, the main examination method for the clinical diagnosis of this disease, is $74-82 \%$ (17). Among the various ultrasonographic features, hypoechogenicity and microcalcification have high specificity for the diagnosis of PTC. Contrast-enhanced ultrasound involves the introduction of an ultrasound contrast agent into the tumor microcirculation to observe tumor vascular distribution and blood perfusion. The main features of using this ultrasound include the centripetal entry of contrast agents, with a slow in and fast out enhancement, heterogeneous low enhancement and larger nodular size compared with that in 2-dimensional grayscale ultrasound.
The aforementioned features of contrast-enhanced ultrasound were utilized in the present study. The distribution of blood and perfusion in PTC nodules are associated with tumor size. The smaller nodules $(<1 \mathrm{~cm})$ may have poor blood flow signals, while larger nodules $(>2 \mathrm{~cm})$ may display the opposite pattern (18-20). Therefore, the present study included tumors $\leq 2 \mathrm{~cm}$ in size. Furthermore, the guidelines for the clinical application of contrast-enhanced ultrasound developed by the European Federation of Societies for Ultrasound in Medicine and Biology (21) still recommend that determination of the results of the contrast-enhanced ultrasound must be performed on 2-dimensional grayscale and color Doppler ultrasound. Therefore, the present study combined the 
Table II. Multivariate logistic regression analysis of ultrasonographic features associated with B-Raf proto-oncogene serine/threonine kinase gene mutation.

\begin{tabular}{lccc}
\hline Factors & OR & $95 \%$ CI & P-value \\
\hline Aspect ratio & 0.612 & $0.179-2.094$ & 0.434 \\
Microcalcification & 2.256 & $1.160-5.500$ & 0.020 \\
Nodule size following & 2.119 & $1.039-4.321$ & 0.039 \\
enhancement & & & \\
Enhancement mode & 1.913 & $0.717-5.099$ & 0.195 \\
Enhancement time & 1.752 & $0.592-5.184$ & 0.311 \\
\hline
\end{tabular}

OR, odds ratio; CI, confidence interval.

features of 2-dimensional grayscale, color Doppler ultrasound and contrast-enhanced ultrasound.

A number of reports indicate a correlation between BRAF gene mutations and ultrasound features, particularly for contrast-enhanced ultrasound, but the results are controversial. Cong et al (22) reported that the BRAFV600E mutation was associated with the blood flow signal in ultrasound, while Kabaker et al (23) identified an association between the BRAFV600E mutation and the boundary, aspect ratio and microcalcification of thyroid nodules upon ultrasound. Sastre-Marcos et al (24) reported an association between the BRAFV600E mutation with at least one of the ultrasound features, and microcalcification and hypoechogenicity were two independently associated factors. Luo et al (25) reported that the BRAFV600E mutation was associated with the features of the nodules on elastography, but not with the features on 2-dimensional ultrasound. Li et al (26) also reported that the BRAFV600E mutation was not associated with the features of 2-dimensional ultrasound (including tumor size, tumor border, or calcification). In the present study, a statistical significant difference was identified between five features of conventional and contrast-enhanced ultrasound, namely, microcalcification, aspect ratio, nodule size following enhancement, enhancement mode and enhancement time, and the BRAFV600E gene mutation status, using univariate logistic regression analysis. Further analysis using multivariate logistic regression revealed that only microcalcification and nodule size following enhancement were significantly associated with BRAF gene mutations $(\mathrm{P}<0.05)$. Previous studies have revealed that hard thyroid nodules are more prone to extravasation and may have a higher degree of relative malignancy $(27,28)$. The hardness of PTC nodules may be derived from their microcalcification, and microcalcification may be associated with BRAF gene mutations. The boundary of the benign nodule is often clear, the nodule size following enhancement is not increased, and its boundary of contrast-enhanced ultrasound is the same as that of 2-dimensional ultrasound. However, the malignant nodule has the nature of invasive growth, and its boundary is often unclear in 2-dimensional ultrasound, therefore the measured size of the nodule using 2-dimensional ultrasound could be smaller than its true size. After contrast-enhanced ultrasound, the boundary can be clearly displayed, which is larger compared with that of 2-dimensional ultrasound (21). Therefore, the increase in nodule size following angiography could be due to the invasive growth of thyroid cancer, and the invasive nature of the tumor may also be an important outcome of BRAF gene mutations.

FNA is suitable for detecting the BRAFV600E mutation (7), but it is an invasive examination. If the nodules are not suitable to determine a diagnosis, further FNA procedures would be required, resulting in increased invasiveness and additional complications, including bleeding. As a non-invasive method, ultrasound is the first choice for thyroid screening (7). The aim of the present study was to determine which characteristic ultrasound feature could reliably be used to screen suspicious BRAF mutations and avoid non-essential FNA. BRAFV600E mutation detection is recommended if the microcalcification and nodule size following enhancement ultrasound features are present, otherwise detection is not recommended. BRAF detection has two advantages: i) Diagnosis: Simple cytological testing has a low positive detection rate for thyroid cancer and is contingent on subjective factors. However, BRAF detection is objective and if a mutation is present, the nodule has an estimated malignancy risk of $>95 \%$ (7); ii) treatment: The 2015 recommendations of the American Thyroid Association indicate that BRAF mutations increase the risk of thyroid cancer recurrence and are associated with other risk factors, such as lymph node metastasis. Aggressive treatment, such as surgery, is recommended for thyroid nodules with BRAF mutations, and patients should be closely monitored if surgery is refused. Observation is recommended if a wild-type BRAF gene is detected (7). Therefore, cytological examination consisting of FNA and BRAF mutation detection will increase the diagnostic rate (2).

The present study identified associations between microcalcification and nodule size following enhancement and the BRAFV600E mutation. If these two features are present, BRAF mutation detection is recommended. Otherwise, BRAF mutation detection should be limited. These criteria provide clinicians with a framework for diagnosis and treatment, and a basis for clinicians and patients to undergo observation. Therefore, ultrasound assessment is a non-invasive method for early PTC screening that may detect BRAF mutations. Following screening, closer follow-up or more aggressive intervention may be required. Even in the case of substantial tissue invasion, following early intervention, the prognosis will be improved.

A limitation to the present study is the small number of patients with PTC included and the fact that the majority of the analysis was qualitative with subjective indexes. Although the conventional and contrast-enhanced ultrasound procedures were performed by experienced sonographers, subjective factors may have affected the data. A number of risk factors can affect the prognosis of PTC, including the BRAF mutation and ultrasound features. Further investigation is required to analyze the clinical course and clinicopathological risk factors of BRAF mutation and ultrasound features. The lymph node dissection was not performed in all of the patients in the present study and the presence of pathological lymph node metastasis in the other patients was unknown. Thus, lymph node conditions should be included to analyze the prognosis of patients in future studies. In addition, patients should be 
followed over a longer postoperative time period to evaluate distant metastasis and/or aggressive tumors that may develop with a positive BRAFV600E mutation status.

In conclusion, the BRAFV600E mutation was associated with microcalcification and nodule size following enhancement. If ultrasound reveals microcalcification or an increase in nodule size following angiography, surgical treatment and BRAF gene detection should be performed.

\section{Acknowledgements}

Not applicable.

\section{Funding}

This study was supported by the National Natural Science Foundation of China (grant no. 81701709), Chongqing Municipal Health and Family Planning Commission (grant no. 2016MSXM029) and Chongqing Science and Technology Commission (grant no. cstc2016jcyjA0295).

\section{Availability of data and materials}

All data generated or analyzed during this study are included in this article.

\section{Authors' contributions}

YL, LH, JC, and LY designed the study and wrote the main manuscript text. YL, LH and GY analyzed all data. GY and LC prepared all figures. LC and BZ performed the FNAB. GY and LY revised the manuscript. All authors read and approved the final manuscript.

\section{Ethics approval and consent to participate}

This retrospective review study was approved by the Ethics Committee of the Second Affiliated Hospital of Chongqing Medical University. The requirement for consent was waived due to the retrospective nature of the study.

\section{Patient consent for publication}

Not applicable.

\section{Competing interests}

The authors declare that they have no competing interests.

\section{References}

1. Du L, Li R, Ge M, Wang Y, Li H, Chen W and He J: Incidence and mortality of thyroid cancer in China, 2008-2012. Chin J Cancer Res 31: 144-151, 2019.

2. Jung CK, Little MP, Lubin JH, Brenner AV, Wells SA Jr, Sigurdson AJ and Nikiforov YE: The increase in thyroid cancer incidence during the last four decades is accompanied by a high frequency of BRAF mutations and a sharp increase in RAS mutations. J Clin Endocrinol Metab 99: E276-E285, 2014.

3. Czarniecka A, Oczko-Wojciechowska M and Barczyński M: BRAF V600E mutation in prognostication of papillary thyroid cancer (PTC) recurrence. Gland Surg 5: 495-505, 2016.
4. Li F, Chen G, Sheng C, Gusdon AM, Huang Y, Lv Z, Xu H, Xing $\mathrm{M}$ and Qu S: BRAFV600E mutation in papillary thyroid microcarcinoma: A meta-analysis. Endocr Relat Cancer 22: 159-168, 2015.

5. Czarniecka A, Kowal M, Rusinek D, Krajewska J, Jarzab M, Stobiecka E, Chmielik E, Zembala-Nozynska E, Poltorak S, Sacher A, et al: The risk of relapse in papillary thyroid cancer (PTC) in the context of BRAFV600E mutation status and other prognostic factors. PLoS One 10: e0132821, 2015.

6. Chen Y, Sadow PM, Suh H, Lee KE, Choi JY, Suh YJ, Wang TS and Lubitz CC: BRAF(V600E) is correlated with recurrence of papillary thyroid microcarcinoma: A systematic review, Multi-institutional primary data analysis, and meta-analysis. Thyroid 26: 248-255, 2016.

7. Haugen BR, Alexander EK, Bible KC, Doherty GM, Mandel SJ, Nikiforov YE, Pacini F, Randolph GW, Sawka AM, Schlumberger M, et al: 2015 American thyroid association management guidelines for adult patients with thyroid nodules and differentiated thyroid cancer: The American thyroid association guidelines task force on thyroid nodules and differentiated thyroid cancer. Thyroid 26: 1-133, 2016.

8. Livak KJ and Schmittgen TD: Analysis of relative gene expression data using real-time quantitative PCR and the 2(-Delta Delta C(T)) method. Methods 25: 402-408, 2001.

9. Brito JP, Ito Y, Miyauchi A and Tuttle RM: A clinical framework to facilitate risk stratification when considering an active surveillance alternative to immediate biopsy and surgery in papillary microcarcinoma. Thyroid 26: 144-149, 2016.

10. Xing M, Alzahrani AS, Carson KA, Viola D, Elisei R, Bendlova B, Yip L, Mian C, Vianello F, Tuttle RM, et al: Association between BRAF V600E mutation and mortality in patients with papillary thyroid cancer. JAMA 309: 1493-1501, 2013.

11. Prescott JD: BRAF V600E status adds incremental value to current risk classification systems in predicting papillary thyroid carcinoma recurrence. Surgery 152: 984-990, 2012.

12. Zheng X, Wei S, Han Y, Li Y, Yu Y, Yun X, Ren X and Gao M: Papillary microcarcinoma of the thyroid: Clinical characteristics and BRAF(V600E) mutational status of 977 cases. Ann Surg Oncol 20: 2266-2273, 2013.

13. Walczyk A, Kowalska A, Kowalik A, Sygut J, Wypiórkiewicz E, Chodurska R, Pięciak L and Góźdź S: The BRAF(V600E) mutation in papillary thyroid microcarcinoma: Does the mutation have an impact on clinical outcome. Clin Endocrinol (Oxf) 80: 899-904, 2014

14. Moon HJ, Kim EK, Chung WY, Shin DY and Kwak JY: BRAF mutation in fine needle aspiration specimens as a potential predictor for persistence/recurrence in patients with classical papillary thyroid carcinoma larger than $10 \mathrm{~mm}$ at a BRAF mutation prevalent area. Head Neck 37: 1432-1438, 2015.

15. Peyssonnaux $\mathrm{C}$ and Eychène $\mathrm{A}$ : The Raf/MEK/ERK pathway: New concepts of activation. Biol Cell 93: 53-62, 2001.

16. Liu C, Chen T and Liu Z: Associations between BRAF(V600E) and prognostic factors and poor outcomes in papillary thyroid carcinoma: A meta-analysis. World J Surg Oncol 14: 241, 2016.

17. Zhang B, Jiang YX, Dai Q, et al: Prospective observation of contrast-enhanced patterns of thyroid nodules with SonoVue. Chin J Med Imaging Technol 26: 844-847, 2010.

18. Yang YJ, Jian-Min L and Wang BC: Study of relationship between VEGF expression and tumor progress of primary hepatocellular carcinoma. China J Modern Med 15: 408-412, 2005.

19. Bartolotta TV, Midiri M, Galia M, Runza G, Attard M, Savoia G, Lagalla $\mathrm{R}$ and Cardinale AE: Qualitative and quantitative evaluation of solitary thyroid nodules with contrast-enhanced ultrasound: Initial results. Eur Radiol 16: 2234-2241, 2006.

20. Zhou Q, Jiang J, Du XP, et al: Value of contrast-enhanced ultrasound in diagnosis of thyroid papillary carcinoma. Chin J Ultrasound Med 27: 595-597, 2011.

21. Claudon M, Cosgrove D, Albrecht T, Bolondi L, Bosio M, Calliada F, Correas JM, Darge K, Dietrich C, D'Onofrio M, et al: Guidelines and good clinical practice recommendations for contrast enhanced ultrasound (CEUS)-update 2008. Ultraschall Med 29: 28-44, 2008.

22. Cong X, Zhao S, Wang Y, et al: Ultrasound features of braf t1799a gene mutation papillary thyroid carcinoma. Med J Qilu 29: 201-212, 2014.

23. Kabaker AS, Tublin ME, Nikiforov YE, Armstrong MJ, Hodak SP, Stang MT, McCoy KL, Carty SE and Yip L: Suspicious ultrasound characteristics predict BRAF V600E-positive papillary thyroid carcinoma. Thyroid 22: 585-589, 2012. 
24. Sastre-Marcos J, Val-Zaballos FD, Cortes-Muñoz C, Campos-Martin Y, Aso-Manso S, Jaen-Diaz I, Vicente-Delgado A and Lopez-Lopez J: BRAF V600E positive papillary thyroid carcinoma is associated with suspicious ultrasound feature. Endocrine 37: EP850, 2015.

25. Luo ZY, Hong YR, Wen Q, et al: Associations of the BRAF V600E mutation with sonographic features in patients with papillary thyroid carcinoma. Chin J Ultrasonogr 25: 785-789, 2016.

26. Li Q, Yuan J, Wang Y and Zhai Y: Association between the BRAF V600E mutation and ultrasound features of the thyroid in thyroid papillary carcinoma. Oncol Lett 14: 1439-1444, 2017.
27. Moon HJ, Kim EK, Yoon JH and Kwak JY: Clinical implication of elastography as a prognostic factor of papillary thyroid microcarcinoma. Ann Surg Oncol 19: 2279-2287, 2012.

28. Jin ZQ, Lin MY, Hu WH, Li WY and Bai SJ: Gray-Scale ultrasonography combined with elastography imaging for the evaluation of papillary thyroid microcarcinoma: As a prognostic clinicopathology factor. Ultrasound Med Biol 40: 1769-1777, 2014.

(c) (i) () This work is licensed under a Creative Commons Attribution-NonCommercial-NoDerivatives 4.0 International (CC BY-NC-ND 4.0) License. 